\title{
Topological superfluid phases of an atomic Fermi gas with in- and out-of-plane Zeeman fields and equal Rashba-Dresselhaus spin-orbit coupling
}

\author{
M. Iskin ${ }^{1}$ and A. L. Subaş ${ }^{2}$ \\ ${ }^{1}$ Department of Physics, Koç University, Rumelifeneri Yolu, 34450 Sartyer, Istanbul, Turkey \\ ${ }^{2}$ Department of Physics, Faculty of Science and Letters, Istanbul Technical University, 34469 Maslak, Istanbul, Turkey
}

(Received 16 November 2012; revised manuscript received 14 May 2013; published 24 June 2013)

\begin{abstract}
We analyze the effects of in- and out-of-plane Zeeman fields on the BCS-Bose-Einstein condensation (BEC) evolution of a Fermi gas with equal Rashba-Dresselhaus (ERD) spin-orbit coupling (SOC). We show that the ground state of the system involves gapless superfluid phases that can be distinguished with respect to the topology of the momentum-space regions with zero excitation energy. For the BCS-like uniform superfluid phases with zero center-of-mass momentum, the zeros may correspond to one or two doubly degenerate spheres, two or four spheres, two or four concave spheroids, or one or two doubly degenerate circles, depending on the combination of Zeeman fields and SOC. Such changes in the topology signal a quantum phase transition between distinct superfluid phases and leave their signatures on some thermodynamic quantities. We also analyze the possibility of Fulde-Ferrell-Larkin-Ovchinnikov (FFLO)-like nonuniform superfluid phases with finite centerof-mass momentum and obtain an even richer phase diagram.
\end{abstract}

DOI: $10.1103 /$ PhysRevA.87.063627

PACS number(s): 67.85.-d, 03.75.Hh, 03.75.Ss, 05.30.Fk

\section{INTRODUCTION}

While topological matter and related phenomena have become a popular subject in the past few years within the condensed-matter community [1-3], a window has recently been opened to the exotic world of topological phases in atomic and molecular physics communities, with arguably a greater premise on a route toward studying them under highly controllable atomic settings [4-7]. The current advances in atomic systems offer the possibility of engineering both Abelian and non-Abelian artificial gauge fields on demand, by coupling the internal states of atoms to their center-of-mass motion via Raman dressing of atomic hyperfine states with laser fields. While there are a number of theoretical proposals for implementing atomic gases with various spin-orbit coupling (SOC) symmetries, several experimental groups have so far only achieved an Abelian equal Rashba-Dresselhaus (ERD) coupling, first with Bose [4,5] and then with Fermi [6,7] gases. Allured by the experimental possibilities, there has been a growing interest in studying spin-orbit-coupled atomic Bose and Fermi gases as functions of the tunable laser parameters including the strength and symmetry of the SOC field, $s$-wave scattering length, and Zeeman fields. Both the uniform and trapped systems have been considered in all one, two, and three dimensions, already revealing exotic single-, two-, few-, and many-body properties at both zero and finite temperatures [8-21].

In regard to the Fermi gases, depending on both the strength and the symmetry of the SOC field, it has been shown that the interplay between the SOC and out-of-plane Zeeman fields gives rise to a variety of topologically nontrivial and thermodynamically stable gapless superfluid phases [12-21]. In confined geometries, some of these phases have been shown to feature Majorana zero-energy bound states, for which the associated quasiparticle operators are self-Hermitian, i.e., a Majorana quasiparticle is its own antiquasiparticle [21]. These quasiparticles play a key role in fault-tolerant quantum computation, and although they are predicted to be found in strongly correlated systems in many fields of physics, including the fractional quantum Hall systems [22], chiral two-dimensional $p$-wave superconductors [23,24] and superfluids [25,26], three-dimensional topological insulator-superconductor heterostructures [27], one-dimensional nanowires [28,29], and spin-orbit-coupled (SOC) semiconductor-superconductor heterostructures [30], their realization has proved to be quite difficult.

Given that the cold atoms offer unprecedented control in comparison to the condensed-matter ones, there is a good chance of creating and observing Majorana quasiparticles with atomic systems in the near future. Motivated by these recent developments [6,7], here we analyze the effects of not only the out-of-plane but also the in-plane Zeeman fields on the phase diagram of a Fermi gas with ERD coupling. Since the inclusion of an in-plane Zeeman field complicates the many-body problem considerably even at the mean-field level, this term has been completely ignored in the recent literature [12-21], even though it appears naturally in the active experiments [6,7]. By including it here, we find gapless superfluid phases that can be distinguished with respect to the topology of the momentum-space regions with zero excitation energy. For instance, for the BCS-like uniform superfluid phases with zero center-of-mass momentum, the zeros may correspond to one or two doubly degenerate spheres, two or four spheres, two or four concave spheroids, or one or two doubly degenerate circles (see Fig. 1). These phases are distinct because even though their order parameters all have the same $s$-wave symmetry, changes in the topology leave strong signatures on some of the thermodynamic quantities, signaling a topological quantum phase transition. We also analyze the possibility of FFLO-like nonuniform superfluid phases with finite center-of-mass momentum [31,32] and obtain an even richer phase diagram.

The rest of the paper is organized as follows. We introduce the Hamiltonian in Sec. II and derive a complete set of nonlinearly coupled self-consistency equations for the 


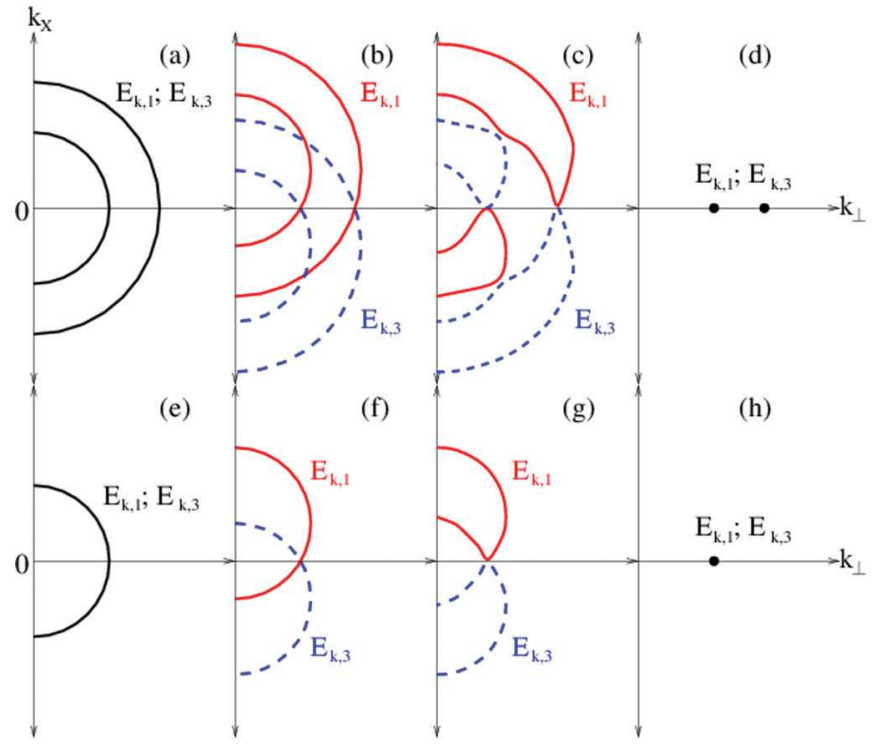

FIG. 1. (Color online) The gapless superfluid phases can be classified with respect to the topology of their momentum-space regions with zero excitation energy. The top (bottom) figures illustrate the possible BCS-like uniform superfluid phases on the BCS (BEC) side, where the zero-energy regions correspond to $[(\mathrm{a}),(\mathrm{e})]$ two (one) doubly degenerate spheres centered at $\mathbf{k}=\mathbf{0}$ when $\alpha=0, h_{O} \neq 0$, and/or $h_{I} \neq 0$; [(b), (f)] four (two) spheres centered at $k_{x}= \pm M \alpha$ and $k_{y}=k_{z}=0$ when $\alpha \neq 0, h_{O}=0$, and $h_{I} \neq 0$; [(c), (g)] four (two) concave spheroids when $\alpha \neq 0, h_{O} \neq 0$, and $h_{I} \neq 0$; and [(d), (h)] two (one) doubly degenerate circles centered at $k_{x}=0$ when $\alpha \neq 0, h_{O} \neq 0$, and $h_{I}=0$. Here, $\alpha, h_{O}$ and $h_{I}$ are the strengths of the SOC, out-of-plane and in-plane Zeeman fields, respectively.

amplitude of the superfluid order parameter, center-of-mass momentum, total number, and polarizations. We solve the resultant equations numerically and obtain the phase diagram of the system for the BCS-like uniform superfluid phases in Sec. III and FFLO-like nonuniform superfluid phases in Sec. IV. A brief summary of our main findings is given in Sec. V.

\section{MEAN-FIELD THEORY}

The results mentioned above are obtained by considering the following fields: the ERD coupling $-\alpha k_{x} \sigma_{y}$ which corresponds to a momentum-dependent Zeeman field in the in-plane $y$ direction, out-of-plane Zeeman field $-h_{O} \sigma_{z}$, and in-plane Zeeman field $-h_{I} \sigma_{y}$, where $\left\{\alpha, h_{O}, h_{I}\right\} \geqslant 0$ are their strengths and $k_{x}$ is the $x$ component of the momentum. These fields have very different origins in cold-atom systems, where the Raman coupling and detuning between the two laser beams driving the Raman transition from the two-photon resonance correspond to the out-of-plane and in-plane Zeeman fields, respectively, and the ERD coupling is the Doppler shift an atom experiences as it moves in the two laser fields [4-7].

The mean-field Hamiltonian for this system (in units of $\hbar=$ $\left.1=k_{B}\right)$ can be written as $H=H_{0}+(1 / 2) \sum_{\mathbf{k}} \psi_{\mathbf{k} \mathbf{Q}}^{\dagger} \mathbf{D}_{\mathbf{k} \mathbf{Q}} \psi_{\mathbf{k} \mathbf{Q}}$, where $H_{0}=\sum_{\mathbf{k}} \xi_{-\mathbf{k}_{-}}+\left|\Delta_{\mathbf{Q}}\right|^{2} / g$, and the matrix $\mathbf{D}_{\mathbf{k} \mathbf{Q}}$ is given by

$$
\left(\begin{array}{cccc}
\xi_{\mathbf{k}_{+}}-h_{O} & S_{\mathbf{k}_{+}}+i h_{I} & 0 & \Delta_{\mathbf{Q}} \\
S_{\mathbf{k}_{+}}^{*}-i h_{I} & \xi_{\mathbf{k}_{+}}+h_{O} & -\Delta_{\mathbf{Q}} & 0 \\
0 & -\Delta_{\mathbf{Q}}^{*} & -\xi_{-\mathbf{k}_{-}}+h_{O} & -S_{-\mathbf{k}_{-}}^{*}+i h_{I} \\
\Delta_{\mathbf{Q}}^{*} & 0 & -S_{-\mathbf{k}_{-}}-i h_{I} & -\xi_{-\mathbf{k}_{-}}-h_{O}
\end{array}\right)
$$

Here, $\quad \psi_{\mathbf{k} \mathbf{Q}}^{\dagger}=\left[a_{\mathbf{k}_{+}, \uparrow}^{\dagger}, a_{\mathbf{k}_{+}, \downarrow}^{\dagger}, a_{-\mathbf{k}_{-}, \uparrow}, a_{-_{-} \mathbf{k}_{-}, \downarrow}\right]$ denotes the fermionic operators collectively where $\mathbf{k}_{ \pm}=\mathbf{k} \pm \mathbf{Q} / \mathbf{2}, \mathbf{Q}=$ $\left(Q_{x}, Q_{y}, Q_{z}\right)$ is the center-of-mass momentum of the Cooper pairs, $\xi_{\mathbf{k}}=\epsilon_{\mathbf{k}}-\mu$ is the shifted dispersion with $\epsilon_{\mathbf{k}}=k^{2} /(2 M)$ and $\mu$ the chemical potential, $\Delta_{\mathbf{Q}}=g\left\langle a_{\mathbf{k}_{+}, \uparrow} a_{-\mathbf{k}_{-}, \downarrow}\right\rangle$ is the mean-field order parameter with $g$ being the strength of the contact interaction and $\langle\cdots\rangle$ denoting the thermal average, and $S_{\mathbf{k}}=i \alpha k_{x}$. We consider both BCS-like uniform superfluid phases with $\Delta_{\mathbf{Q}}=\Delta_{0}$, and FFLO-like nonuniform superfluid phases with $\Delta_{\mathbf{Q}}=\left|\Delta_{0}\right| e^{i \mathbf{Q} \cdot \mathbf{R}}$, where $\mathbf{R}$ is the center-of-mass position. We eliminate $g$ in favor of the $s$-wave scattering length $a_{s}$ via the relation $1 / g=-M V /\left(4 \pi a_{s}\right)+\sum_{\mathbf{k}} 1 /\left(2 \epsilon_{\mathbf{k}}\right)$, where $V$ is the volume. The thermodynamic potential for this Hamiltonian is given by $\Omega=H_{0}+(T / 2) \sum_{\mathbf{k}, \lambda} \ln \left[1-f\left(E_{\mathbf{k} \mathbf{Q}, \lambda}\right)\right]$, where $T$ is the temperature, $\lambda=\{1,2,3,4\}$ labels the quasiparticle/quasihole excitation energies $E_{\mathbf{k Q}, 1}=-E_{-\mathbf{k} \mathbf{Q}, 3}$ and $E_{\mathbf{k} \mathbf{Q}, 2}=-E_{-\mathbf{k} \mathbf{Q}, 4}$ as determined by the eigenvalues of the matrix given above, and $f(x)=1 /\left(e^{x / T}+1\right)$ is the Fermi function.

The self-consistency equations for $\left|\Delta_{0}\right|$ and $Q_{i}$ are obtained by minimizing $\Omega$, i.e., $\partial \Omega / \partial\left|\Delta_{0}\right|=0$ and $\partial \Omega / \partial Q_{i}=0$ for $i \equiv(x, y, z)$, respectively, and this procedure leads to

$$
\begin{gathered}
\frac{\left|\Delta_{0}\right|}{g}=-\frac{1}{4} \sum_{\mathbf{k}, \lambda} \frac{\partial E_{\mathbf{k} \mathbf{Q}, \lambda}}{\partial\left|\Delta_{0}\right|} f\left(E_{\mathbf{k} \mathbf{Q}, \lambda}\right), \\
Q_{i}=-\frac{2 \pi^{3} M}{k_{0}^{3} V} \sum_{\mathbf{k}, \lambda} \frac{\partial E_{\mathbf{k} \mathbf{Q}, \lambda}}{\partial Q_{i}} f\left(E_{\mathbf{k} \mathbf{Q}, \lambda}\right) .
\end{gathered}
$$

Here, while the momentum-space cutoff $k_{0}$ used in the numerical evaluation of $\mathbf{k}$-space integrations appears explicitly in Eq. (3), due to the term $\sum_{\mathbf{k}} 1=V\left(k_{0} / \pi\right)^{3}$ in three dimensions where $-k_{0} \leqslant k_{i} \leqslant k_{0}$ for $i=\{x, y, z\}$, we checked that our results do not depend on its specific value as long as $k_{0}$ is sufficiently large compared to the Fermi momentum $k_{F}=\left(3 \pi^{2} N / V\right)^{1 / 3}$. In addition, we obtain the total number of particles and in- and out-of-plane number polarizations via $N=-\partial \Omega / \partial \mu$ and $P_{I, O}=-(1 / N) \partial \Omega / \partial h_{I, O}$, leading to

$$
\begin{gathered}
N=\frac{1}{4} \sum_{\mathbf{k}, \lambda}\left[1-2 \frac{\partial E_{\mathbf{k} \mathbf{Q}, \lambda}}{\partial \mu} f\left(E_{\mathbf{k} \mathbf{Q}, \lambda}\right)\right], \\
P_{I}=-\frac{1}{2 N} \sum_{\mathbf{k}, \lambda} \frac{\partial E_{\mathbf{k} \mathbf{Q}, \lambda}}{\partial h_{I}} f\left(E_{\mathbf{k} \mathbf{Q}, \lambda}\right), \\
P_{O}=-\frac{1}{2 N} \sum_{\mathbf{k}, \lambda} \frac{\partial E_{\mathbf{k} \mathbf{Q}, \lambda}}{\partial h_{O}} f\left(E_{\mathbf{k} \mathbf{Q}, \lambda}\right) .
\end{gathered}
$$

We also use these expressions to extract the projections $n_{\mathbf{k}, \delta}$ of the momentum distribution along the out-of-plane $z$ direction where $\delta=(\uparrow, \downarrow)$, and in-plane $y$ and $x$ directions where $\delta=(\rightarrow, \leftarrow)$ and $\delta=(\swarrow, \nearrow)$, respectively. These seven equations given in Eqs. (2)-(6) correspond to our self-consistency equations at the mean-field level. In the 
forthcoming Secs. III and IV, we solve them to obtain the phase diagrams of the system for the BCS- and FFLO-like superfluid phases, respectively.

\section{BCS-LIKE UNIFORM SUPERFLUID PHASES}

To analyze the BCS-like uniform superfluid phases, it is sufficient to solve four equations only, i.e., Eqs. (2), (4), (5), and (6), with $\mathbf{Q}=\mathbf{0}$, for a self-consistent set of $\left|\Delta_{0}\right|, \mu, h_{I}$, and $h_{O}$ values. We also checked the stability of our meanfield solutions for the uniform superfluid phase using the curvature criterion, i.e., a nonuniform superfluid phase (e.g., a phase separation) is favored when $\partial^{2} \Omega / \partial\left|\Delta_{0}\right|^{2}<0$, since this criterion coincides with that of the compressibility one, i.e., the matrix $\kappa_{\sigma \sigma^{\prime}}=-\partial^{2} \Omega /\left(\partial \mu_{\sigma} \partial \mu_{\sigma^{\prime}}\right)$ must be positive definite for the stability of the obtained solutions. Before we present the resultant phase diagrams, let us first analyze the excitation spectrum of the system.

\section{A. Excitation spectrum}

In general, all four branches of the excitation spectrum may be different from each other, and hence both $E_{\mathbf{k} \mathbf{0}, \lambda}$ and their $\left|\Delta_{0}\right|, \mu, h_{I}$ and $h_{O}$ derivatives are not analytically tractable and need to be evaluated simultaneously with the self-consistency Eqs. (2) and (4) for all k-space points. This makes the current work numerically more demanding compared to the previous ones [12-20], for which the explicit forms of $E_{\mathbf{k} \mathbf{0}, \lambda}$ can be found. In order to understand the topology of the resultant superfluid phases, next we analyze the analytically tractable limits.

\section{No SOC limit}

In the absence of a SOC, setting $\mathbf{Q}=\mathbf{0}$ and $\alpha=0$ in Eq. (1), we obtain

$$
E_{\mathbf{k} \mathbf{0}, \lambda}=p_{\lambda} h_{T}+s_{\lambda} \sqrt{\xi_{\mathbf{k}}^{2}+\left|\Delta_{0}\right|^{2}},
$$

where $s_{1}=s_{2}=p_{2}=p_{3}=+1$ and $s_{3}=s_{4}=p_{1}=p_{4}=$ -1 and $h_{T}=\sqrt{h_{O}^{2}+h_{I}^{2}}$ is the strength of the total Zeeman field, showing that $E_{\mathbf{k} \mathbf{0}, 1}=-E_{\mathbf{k} \mathbf{0}, 3}$ can have zero-energy regions in $\mathbf{k}$ space. The doubly degenerate zeros are determined by $h_{T}^{2}-\xi_{\mathbf{k}}^{2}=\left|\Delta_{0}\right|^{2}$, and when $h_{T}>\left|\Delta_{0}\right|$ these conditions give two spheres of zeros for $\mu>\sqrt{h_{T}^{2}-\left|\Delta_{0}\right|^{2}}$ and one sphere of zeros for $\mu<\sqrt{h_{T}^{2}-\left|\Delta_{0}\right|^{2}}$. Therefore, the transition from gapped superfluid to gapless superfluid occurs at $h_{T}=\left|\Delta_{0}\right|$, and $\mu=0$ determines the transition from gapless superfluid with two doubly degenerate spheres of zeros to the one with one doubly degenerate sphere of zeros; i.e., it gives the critical point for the disappearance of the doubly degenerate inner sphere. These possibilities are schematically illustrated in Figs. 1(a) and 1(e), and the corresponding gapless superfluid phases in this case are known to be topologically trivial [12-21].

\section{No out-of-plane Zeeman field limit}

In the absence of an out-of-plane Zeeman field, setting $\mathbf{Q}=\mathbf{0}$ and $h_{O}=0$, we obtain

$$
E_{\mathbf{k} \mathbf{0}, \lambda}=p_{\lambda} h_{I}+s_{\lambda} \sqrt{\left(\xi_{\mathbf{k}}+p_{\lambda} \alpha k_{x}\right)^{2}+\left|\Delta_{0}\right|^{2}}
$$

showing that $E_{\mathbf{k} \mathbf{0}, 1}=-E_{-\mathbf{k} \mathbf{0}, 3}$ can have zeros. The zeros are determined by $\widetilde{k}_{x}^{2}+k_{\perp}^{2}=2 M\left(\tilde{\mu} \pm \sqrt{h_{I}^{2}-\left|\Delta_{0}\right|^{2}}\right)$, where $\tilde{k}_{x}=k_{x}-M \alpha, k_{\perp}=\sqrt{k_{y}^{2}+k_{z}^{2}}$, and $\tilde{\mu}=\mu-M \alpha^{2} / 2$, and when $h_{I}>\left|\Delta_{0}\right|$ these conditions give four spheres of zeros for $\tilde{\mu}>\sqrt{h_{I}^{2}-\left|\Delta_{0}\right|^{2}}$ and two spheres of zeros for $\tilde{\mu}<$ $\sqrt{h_{I}^{2}-\left|\Delta_{0}\right|^{2}}$. Therefore, the transition from gapped superfluid to gapless superfluid occurs at $h_{I}=\left|\Delta_{0}\right|$, and $\tilde{\mu}=0$ determines the transition from gapless superfluid with four spheres of zeros to the one with two spheres of zeros; i.e., it gives the critical point for the simultaneous disappearance of two inner (one each from $E_{\mathbf{k} 0,1}$ and $E_{\mathbf{k} \mathbf{0}, 3}$ ) spheres. The superfluid phase with four spheres of zeros is illustrated in Fig. 2(a). In this figure, the main effect of ERD coupling $-\alpha k_{x} \sigma_{y}$ is best seen in the $y$ projection $n_{\mathbf{k}, \rightarrow}$ and $n_{\mathbf{k}, \leftarrow}$ of the momentum distribution as a $k_{x}$-dependent Zeeman field, since $h_{y} \neq 0$ breaks the time-reversal symmetry for this projection. Note that since the SOC gauge field can be integrated out via a shift in the $k_{x}$ momentum and $\mu$, the corresponding gapless superfluid phases have the same topology and number as case (1), and therefore they are also topologically trivial. However, in comparison to case (1), since the SOC breaks the inversion symmetry in the $k_{x}$ direction, $\alpha \neq 0$ removes most of the degeneracy of zeros except for one or four circles, as schematically illustrated in Figs. 1(b) and 1(f). Note also that sufficiently large $\alpha$ splits these spheres of zeros completely away from each other, leaving no degeneracy.

\section{No in-plane Zeeman field limit}

In the absence of an in-plane Zeeman field, setting $\mathbf{Q}=\mathbf{0}$ and $h_{I}=0$, we obtain

$$
E_{\mathbf{k} \mathbf{0}, \lambda}=s_{\lambda} \sqrt{\xi_{\mathbf{k}}^{2}+h_{O}^{2}+\alpha^{2} k_{x}^{2}+\left|\Delta_{0}\right|^{2}+2 s_{\lambda} p_{\lambda} A_{\mathbf{k}}},
$$

where $A_{\mathbf{k}}=\sqrt{h_{O}^{2}\left(\xi_{\mathbf{k}}^{2}+\left|\Delta_{0}\right|^{2}\right)+\alpha^{2} k_{x}^{2} \xi_{\mathbf{k}}^{2}}$, showing that $E_{\mathbf{k} 0,1}=-E_{\mathbf{k} 0,3}$ can have zeros. The doubly degenerate zeros are determined by $k_{x}=0$ and $h_{O}^{2}-\xi_{\mathbf{k}}^{2}=\left|\Delta_{0}\right|^{2}$, and when $h_{O}>\left|\Delta_{0}\right|$ these conditions give two circles of zeros for $\mu>\sqrt{h_{O}^{2}-\left|\Delta_{0}\right|^{2}}$ and one circle of zeros for $\mu<\sqrt{h_{O}^{2}-\left|\Delta_{0}\right|^{2}}$. Therefore, the transition from gapped superfluid to gapless superfluid occurs at $h_{O}=\left|\Delta_{0}\right|$, and $\mu=0$ determines the transition from gapless superfluid with two doubly degenerate circles of zeros to the one with one doubly degenerate circle of zeros; i.e., it gives the critical point for the disappearance of the doubly degenerate inner circle. These possibilities are shown in Figs. 1(d) and 1(h), and in sharp contrast to the cases (1) and (2), the corresponding gapless superfluid phases in this case are known to be topologically nontrivial $[14,16]$. The superfluid phase with two doubly degenerate circles of zeros is also illustrated in Fig. 2(c), where the main effect of ERD coupling is best seen in the $z$ and $y$ projections of the momentum distribution as a $k_{x}$-dependent Zeeman field. Note here that even though we choose $P_{I} \neq 0$ in this figure, as shown in Fig. 3(c), this particular data has the same k-space topology as the $P_{I}=0$ limit. Therefore, it is for this reason the time-reversal symmetry is broken by both $h_{z} \neq 0$ and $h_{y} \neq 0$, showing up in the $z$ and $y$ projections. 
(a)
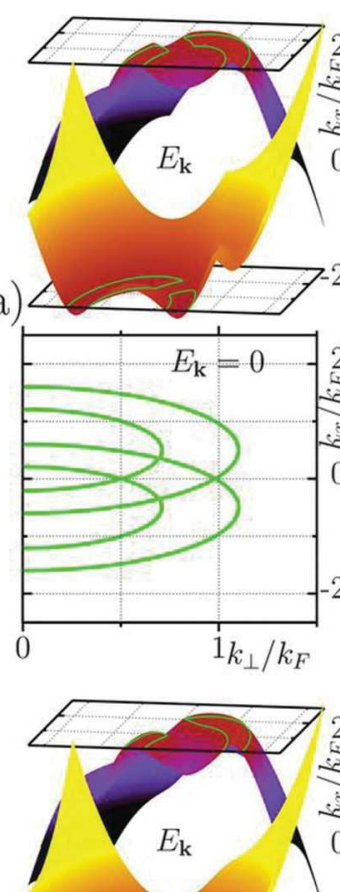

(b)

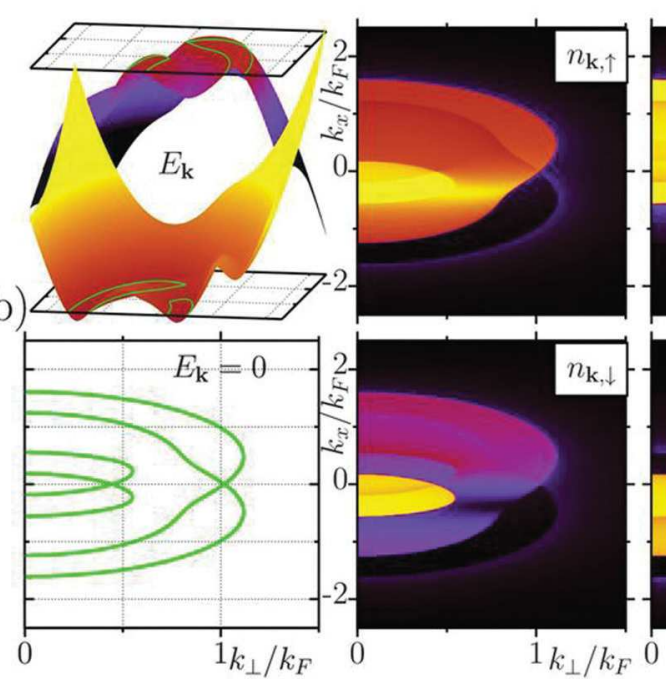

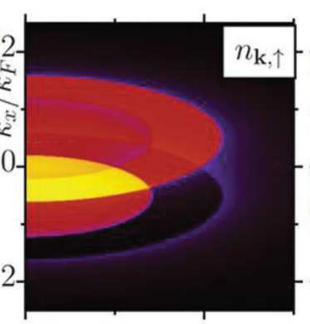
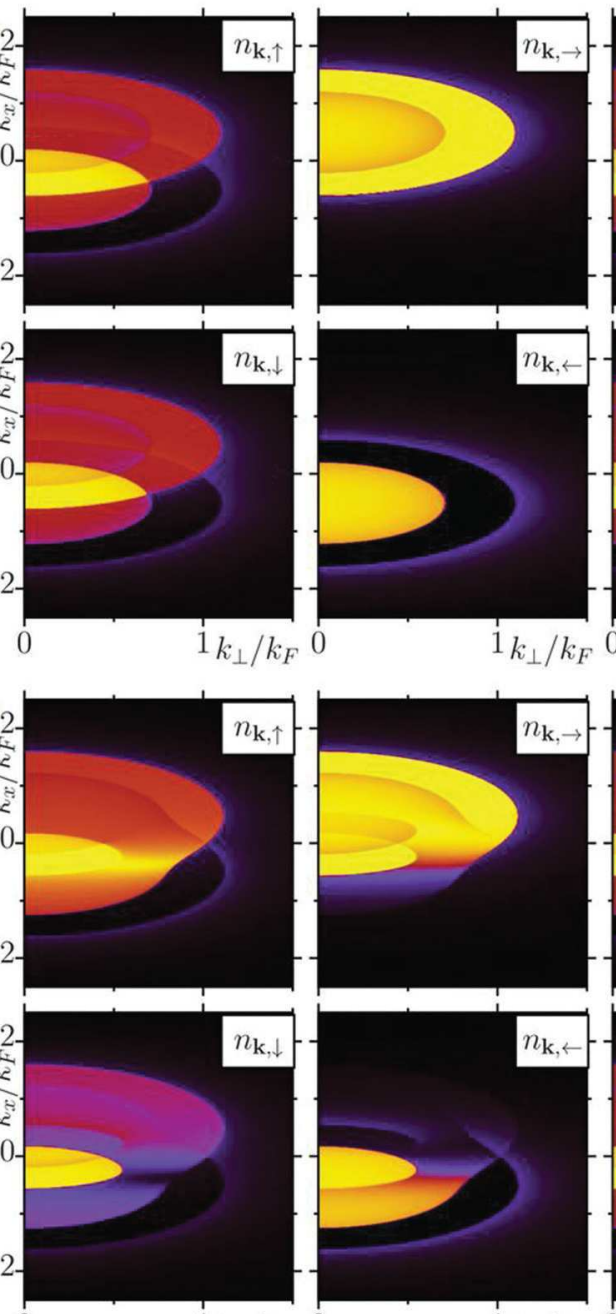

$1 k_{\perp} / k_{F} 0$
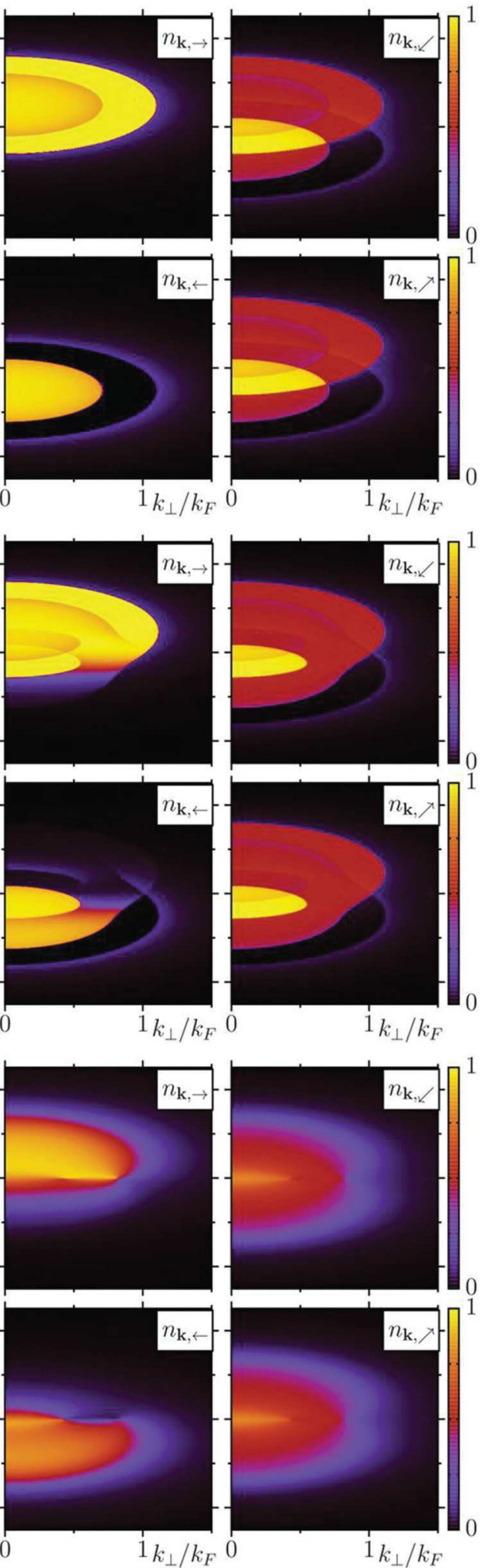

FIG. 2. (Color online) The gapless branches of the energy spectrum $E_{\mathbf{k} \mathbf{0}, 1}$ and $E_{\mathbf{k} \mathbf{0}, 3}$ (in units of $\epsilon_{F}$ ) and the projections of the momentum distribution $n_{\mathbf{k}, \delta}$, where $\delta=(\uparrow, \downarrow)$ for the out-of-plane $z, \delta=(\rightarrow, \leftarrow)$ for the in-plane $y$, and $\delta=(\swarrow, \nearrow)$ for the $x$ direction are shown as a function of $k_{x}$ and $k_{\perp}=\sqrt{k_{y}^{2}+k_{z}^{2}}$ for the BCS-like uniform superfluid phases with (a) four spheres, (b) four concave spheroids, and (c) two doubly degenerate circles of zeros. Here, we set $1 /\left(k_{F} a_{s}\right)=0$ and $\alpha=0.5 k_{F} / M$, and choose $P_{O}=0$ and $P_{I}=0.5$ in panel (a), $P_{O}=0.25$ and $P_{I}=0.5$ in panel (b), and $P_{O}=0.25$ and $P_{I}=0.1$ in panel (c). Note that $E_{\mathbf{k} 0,1}=-E_{-\mathbf{k} 0,3}=0$ regions coincide with the locations of the sharp $n_{\mathbf{k}, \delta}$ features. 


\section{The generic case}

In the light of these limits, it is easier to understand the topology of zeros in the most general case when all three (Zeeman and SOC) gauge fields are nonzero. This generic case is schematically illustrated in Figs. 1(c) and 1(g), showing that $h_{O} \neq 0$ breaks the remaining degeneracy of two circles of zeros (only the ones at finite $k_{x}$ ) that are still present in Fig. 1(b). Therefore, the topology of gapless regions correspond to four concave spheroids on the BCS side and two concave spheroids on the BEC side, and the transition between the two occurs around $\mu \approx 0$, with the simultaneous disappearance of the two inner (one each from $E_{\mathbf{k} 0,1}$ and $E_{\mathbf{k 0}, 3}$ ) concave spheroids. For instance, Fig. 2(b) shows the full $\mathbf{k}$ dependence of the gapless branches of the excitation spectrum and projections of the momentum distribution for the superfluid phase with four concave spheroids of zeros. Note again that the main effect of ERD coupling is best seen in the $z$ and $y$ projections of the momentum distribution as a $k_{x}$-dependent Zeeman field, since the time-reversal symmetry is broken by both $h_{z} \neq 0$ and $h_{y} \neq 0$ for these projections. It is important to emphasize that the exotic superfluid phases discussed above, in particular the ones illustrated in Figs. 1(b), 1(c), 1(f), and 1(g), as well as the numerous possible ways of transitions in between, are unique to this work. To explore the feasibility of observing these phases in cold-atom experiments, next we analyze the phase diagram of the system at $T=0$.

\section{B. Ground-state phase diagrams}

The generic phase diagrams are shown in Fig. 3. We note that, since the SOC can be integrated out of the self-consistency Eqs. (2) and (4) on the $x$ axis when $h_{O}=0$, the critical $h_{I}$ or $P_{I}$ for the transitions from a gapped superfluid to a gapless one with two or four spheres of zeros and from the latter to the normal phase are exactly the same as those obtained for a population-imbalanced Fermi gas without the SOC, i.e., for the transition from a gapped superfluid to a gapless one with one or two doubly degenerate spheres of zeros and from the latter to the normal phase. Here, we locate the normal phase boundaries by finding $\left|\Delta_{0}\right| \lesssim 10^{-3} \epsilon_{F}$, where $\epsilon_{F}=k_{F}^{2} /(2 M)$ is the Fermi energy, and the inaccessible regions are determined by $P_{I}^{2}+$ $P_{O}^{2} \geqslant 1$. In addition, on the $y$ axis when $h_{I}=0$, our results recover the recent works $[14,16]$.

Away from the $x$ and $y$ axes, we find that the gapless superfluid phases with two or four concave spheroids of zeros occupy quite large regions in the phase diagrams as shown in Fig. 3. Depending on $\alpha$ and $a_{s}$, note that the transition from a gapless superfluid phase with two or four spheres of zeros to the one with two or four concave spheroids of zeros may require a finite threshold for $h_{O}$. For instance, when the SOC is weak so that the spheres of zeros contain doubly degenerate circles as shown in Figs. 1(b) and 1(f), an arbitrarily small $h_{O}$ splits these degeneracies, immediately giving rise to the transition. This is clearly seen in Fig. 3(c). In contrast, when the SOC is sufficiently strong so that it splits the spheres of zeros completely away from each other, leaving no degeneracy, the transition occurs at a finite $h_{O}$, as shown in Figs. 3(a) and 3(b).

Next we note an important difference between the grandcanonical (Zeeman fields) and canonical (number polarizations) phase diagrams on the BCS side of the BCS-BEC
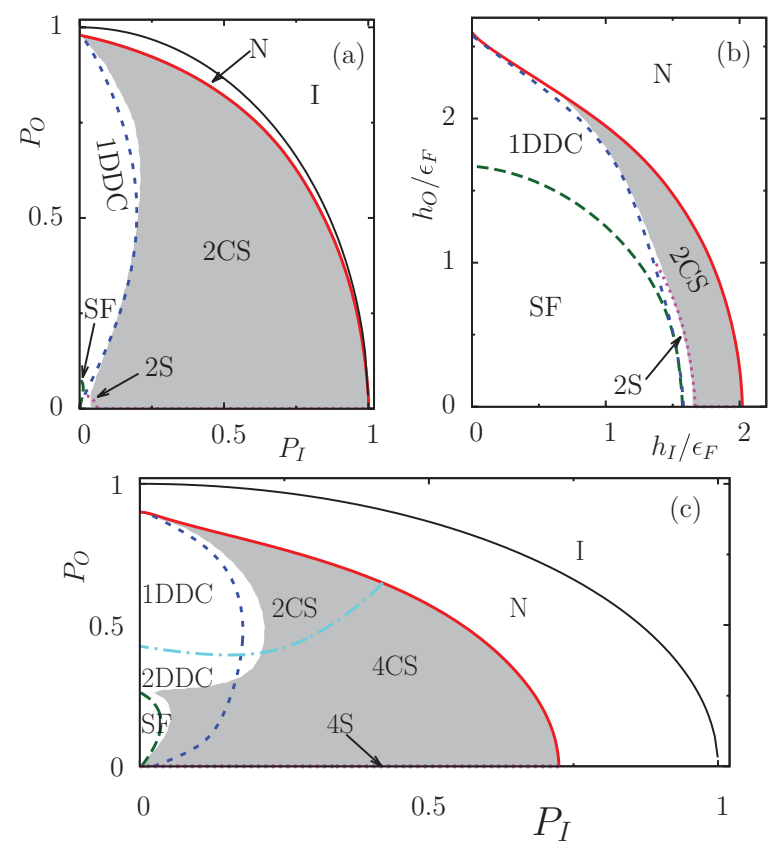

FIG. 3. (Color online) The ground-state phase diagram is shown for the BCS-like uniform superfluid phases as a function of out-ofplace and in-plane [(a), (c)] number polarizations and (b) Zeeman fields, where $1 /\left(k_{F} a_{s}\right)=1$ in panels (a) and (b) and 0 in panel (c). Here, $\alpha=0.5 k_{F} / M$ in all figures, and the labels correspond to an inaccessible region (I), normal (N) phase, gapped superfluid (SF), and gapless superfluid with zeros of one or two doubly degenerate circles (1DDC, 2DDC), two or four spheres (2S, 4S), or two or four concave spheroids (2CS, 4CS). The curvature criterion is $\partial^{2} \Omega / \partial\left|\Delta_{0}\right|^{2}<0$ in the shaded region, indicating an instability toward a nonuniform superfluid phase.

evolution and consider the $h_{O}=0$ limit for the sake of its simplicity. In a grand-canonical phase diagram, where $h_{I}$ is increased from 0 , it can be shown that while $\left|\Delta_{0}\right|$ is unaffected by $h_{I} \neq 0$ until $h_{I}=\left|\Delta_{00}\right|$, where $\left|\Delta_{00}\right|$ is the value of the field-free order parameter when $h_{I}=0$, it immediately vanishes for $h_{I}>\left|\Delta_{00}\right|$. Therefore, increasing $h_{I}$ leads to a first-order quantum phase transition between a gapped superfluid and normal phase, without any intermediate gapless superfluid phase in between. On the other hand, in the case of a canonical phase diagram, where $P_{I}$ is increased from 0 , there exists a $\left|\Delta_{0}\right| \neq 0$ solution with $h_{c}<h_{I}<\left|\Delta_{00}\right|$, where $h_{c}$ is determined by the maximum possible number polarization $P_{I}=P_{c}$. Therefore, in sharp contrast to the grand-canonical case, it is possible to obtain a gapless superfluid phase with increasing $P_{I}$. For this reason, the canonical phase diagram suits better for presenting the possible gapless superfluid phases at unitarity as shown in Fig. 3(c). However, on the BEC side of the BCS-BEC evolution, such a complication does not exist, and it is possible to map the grand-canonical and canonical phase diagrams onto each other as shown in Figs. 3(a) and 3(b).

We emphasize that even though all of these gapless superfluid phases have order parameters with the same $s$ wave symmetry, they can be classified with respect to the topology of their zeros. It is well known in other contexts that such changes in the topology leave strong evidence on the 
thermodynamic quantities such as the atomic compressibility, spin susceptibility, and momentum distribution, signaling a topological quantum phase transition. For instance, in nodal $p$-wave superconductors and superfluids for which the order parameters are $\mathbf{k}$ dependent, while the excitation spectrum $E_{\mathbf{k}}=\sqrt{\xi_{\mathbf{k}}^{2}+\left|\Delta_{\mathbf{k}}\right|^{2}}$ has gapless points of zeros in $\mathbf{k}$ space (which are determined by the nodes of $\left|\Delta_{\mathbf{k}}\right|=0$ ) when $\mu>0$ on the BCS side, it is fully gapped when $\mu<0$ on the BEC side, showing a Lifshitz-type phase transition in the BCS-BEC evolution which occurs precisely at $\mu=0[23,24]$. The presented phase diagrams clearly demonstrate here that it is possible to simulate a much richer variety of gapless superfluid phases and numerous possible ways of phase transitions between them with active experiments. Next, we investigate whether these phases survive if we allow a finite center-of-mass momentum $\mathbf{Q}$.

\section{FFLO-LIKE NONUNIFORM SUPERFLUID PHASES}

In the previous section, having shown that the presence of an in-plane Zeeman field has a dramatic effect on the stability of BCS-like uniform superfluid phases with $\mathbf{Q}=\mathbf{0}$, i.e., nonuniform superfluid phases occupy a very large region in the phase diagrams when $P_{I} \neq 0$, here we discuss the possibility of FFLO-like nonuniform (spatially modulated) superfluid phases with $\mathbf{Q} \neq \mathbf{0}$ as a possible ground state of the system such that $\Delta_{\mathbf{Q}}=\left|\Delta_{0}\right| e^{i \mathbf{Q} \cdot \mathbf{R}}$ (see Sec. II). For this purpose, one needs to solve all seven equations, i.e., Eqs. (2)-(6), in general, for a self-consistent set of $\left|\Delta_{0}\right|, Q_{x}, Q_{y}, Q_{z}, \mu, h_{I}$, and $h_{O}$ values, which is a highly nontrivial task. Our numerical solutions suggest that $Q_{x} \gg\left\{Q_{y}, Q_{z}\right\} \rightarrow 0$ for a greater portion of the parameter space, and therefore, we set $Q_{y}=Q_{z}=0$ and solve only for $Q_{x}$.

In Fig. 4, we show the color maps of the magnitude of the order parameter $\left|\Delta_{0}\right|$ and $x$ component $Q_{x}$ of the center-of-mass momentum, together with the ground-state phase diagram when $1 /\left(k_{F} a_{s}\right)=0$ and $\alpha=0.5 k_{F} / M$. These parameters correspond to that of the figure shown in Fig. 3(c). First of all, we find that $Q_{x}=0$ on the $P_{O}$ axis when $P_{I}=0$ and therefore conclude that FFLO-like nonuniform superfluid phases are not favored in the absence of an in-plane Zeeman field, which is consistent with the stability analysis given in Fig. 3(c). On the other hand, we find that $Q_{x}$ grows almost linearly on the $P_{I}$ axis, i.e., $Q_{x} / k_{F} \simeq P_{I}$, and the FFLO-like phases are strongly favored in the absence of an out-of-plane Zeeman field, which is again consistent with the stability analysis given in Fig. 3(c). Therefore, there is a clear competition between out-of-plane and in-plane Zeeman fields when both fields are present. It turns out that the effects of the in-plane field rapidly dominate over the out-of-plane one, as a result of which FFLO-like phases occupy almost the entire phase diagram. In addition, a direct comparison between Figs. 3(c) and 4(c) shows that FFLO-like phases are more favored than the BCS-like ones against the normal phase.

We note that while allowing the superfluid order parameter to have a single nonzero center-of-mass momentum $\mathbf{Q}$ favors the nonuniform phase over the uniform one where $\mathbf{Q}=\mathbf{0}$, this may not be the case if we allow multiple $\mathbf{Q}$ momenta, e.g., $\Delta_{\mathbf{Q}}=\left|\Delta_{0}\right| \cos (\mathbf{Q} \cdot \mathbf{R})$. This is because FFLO-like
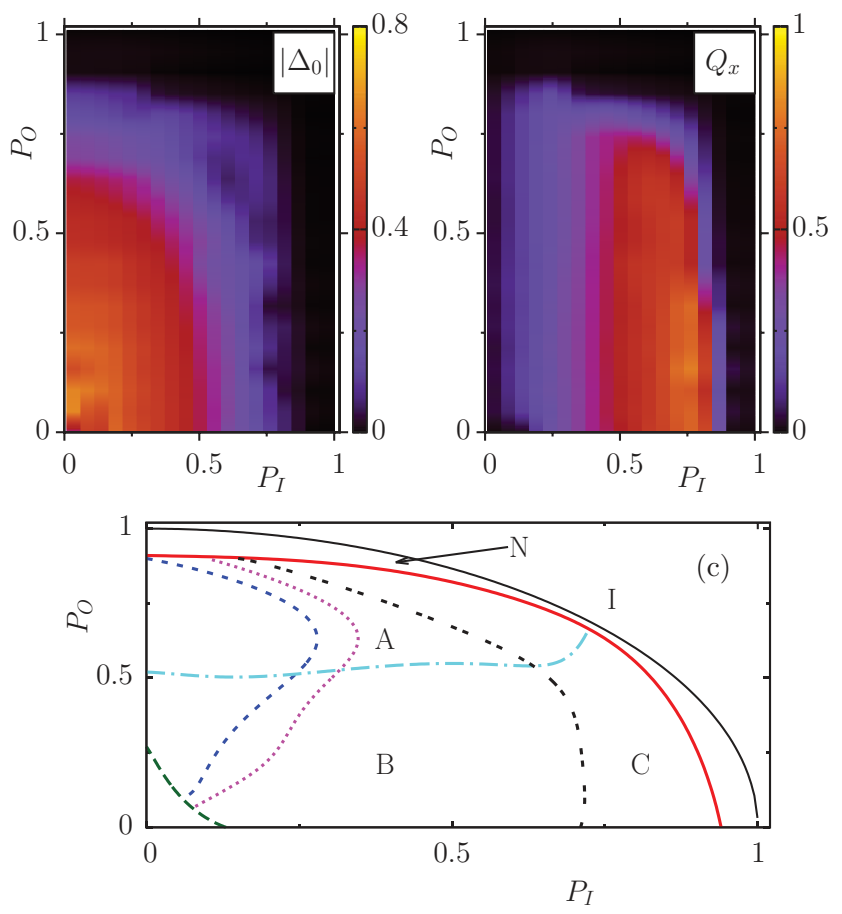

FIG. 4. (Color online) The color maps of the magnitude $\left|\Delta_{0}\right|$ (in units of $\left.\epsilon_{F}\right)$ of the order parameter $\Delta_{\mathbf{Q}}=\left|\Delta_{0}\right| e^{i \mathbf{Q} \cdot \mathbf{R}}$ and $x$ component $Q_{x}$ (in units of $k_{F}$ ) of the center-of-mass momentum and the ground-state phase diagram are shown for the FFLO-like nonuniform superfluid phases, where we set $1 /\left(k_{F} a_{s}\right)=0$ and $\alpha=0.5 k_{F} / M$, i.e., same as in Fig. 3(c). The FFLO-like phases are further classified with respect to the topology of the momentum-space regions with zero excitation energy in panel (c). The $A, B$, and $C$ regions are further illustrated in Fig. 5.

nonuniform superfluid phases in spin-orbit-coupled Fermi gases are stabilized mainly by the asymmetry of the Fermi surfaces in $\mathbf{k}$ space, and this mechanism is in contrast with that of the condensed-matter ones where they are stabilized by the symmetric Zeeman mismatch in $\mathbf{k}$ space. Therefore, only in the latter case, we expect the solutions involving the superpositions of $\mathbf{Q}$ and $-\mathbf{Q}$ momenta to be favored even more than the $+\mathbf{Q}$ or $-\mathbf{Q}$ solutions themselves. However, such a comparison is beyond the scope of this work.

In addition, similar to our analysis for the BCS-like uniform superfluid phases given above in Sec. III, we further classify the FFLO-like nonuniform superfluid phases with respect to the topology of the momentum-space regions with zero excitation energy. Although the zeros of some of the phases shown in Fig. 4(c) are very similar to the BCS-like ones shown in Figs. 1 and $3(\mathrm{c})$, there are also a variety of new ones arising due solely to $\mathbf{Q} \neq 0$. For instance, in Fig. 5, we illustrate the gapless branches of the excitation spectrum as well as the projections of the momentum distribution $n_{\mathbf{k}, \delta}$ for three of the topologically distinct phases labeled in Fig. 4(c). Unlike the case of BCS-like superfluid phases shown in Fig. 2, note that the particle-hole symmetry $E_{\mathbf{k Q}, 1}=-E_{-\mathbf{k Q}, 3}=0$ does not necessarily require the zero-energy regions of the FFLO-like superfluids to have the inversion symmetry around $k_{x}=0$. In addition, we see that the main effect of ERD coupling is best seen in the $z$ and $y$ projections of the momentum distribution as a 

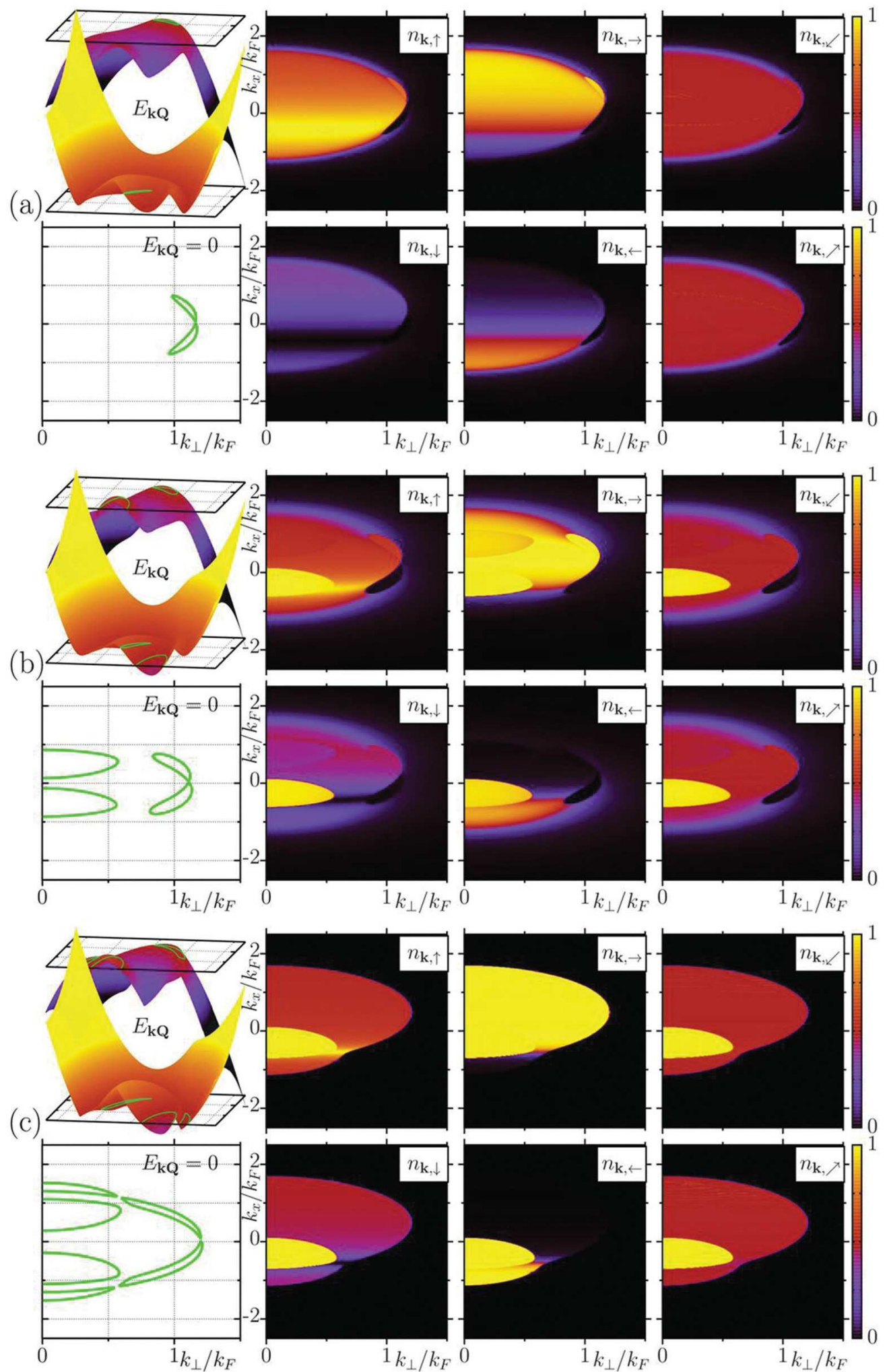

FIG. 5. (Color online) The gapless branches of the energy spectrum $E_{\mathbf{k Q}, 1}$ and $E_{\mathbf{k Q}, 3}$ (in units of $\epsilon_{F}$ ) and the projections of the momentum distribution $n_{\mathbf{k}, \delta}$, where $\delta=(\uparrow, \downarrow)$ for the out-of-plane $z, \delta=(\rightarrow, \leftarrow)$ for the in-plane $y$, and $\delta=(\swarrow, \nearrow)$ for the $x$ direction, are shown as a function of $k_{x}$ and $k_{\perp}=\sqrt{k_{y}^{2}+k_{z}^{2}}$ for the FFLO-like nonuniform superfluid phases. Here, we set $1 /\left(k_{F} a_{s}\right)=0$ and $\alpha=0.5 k_{F} / M$, and choose $P_{O}=0.6$ and $P_{I}=0.5$ in panel (a), $P_{O}=0.2$ and $P_{I}=0.5$ in panel (b), and $P_{O}=0.1$ and $P_{I}=0.75$ in panel (c), and they represent, respectively, the typical topology of $A, B$, and $C$ regions of Fig. 4 . Note that $E_{\mathbf{k Q}, 1}=-E_{-\mathbf{k Q}, 3}=0$ regions and the locations of the sharp $n_{\mathbf{k}, \delta}$ features are shifted by $\mathbf{Q} / 2$. 
$k_{x}$-dependent Zeeman field, since the time-reversal symmetry is broken by both $h_{z} \neq 0$ and $h_{y} \neq 0$ for these projections. For FFLO-like superfluid phases, this figure clearly shows that sharp features of $n_{\mathbf{k}, \delta}$ and zero-excitation-energy regions $E_{\mathbf{k} \mathbf{Q}, 1}=-E_{-\mathbf{k Q}, 3}=0$ do not match in $\mathbf{k}$ space, and they are shifted exactly by $\mathbf{Q} / 2$, i.e., $Q_{x} / 2$, in our paper. These shifts are important since it is in sharp contrast to the BCS-like superfluid phases shown in Fig. 2, where sharp features of $n_{\mathbf{k}, \delta}$ and zero-excitation-energy regions exactly coincide in $\mathbf{k}$ space. Therefore, the competing BCS-like gapless uniform and FFLO-like nonuniform superfluid ground states can easily be distinguished by directly looking at their momentum distributions, which has probably been the most commonly used probing technique in cold atom systems since the early days.

\section{v. CONCLUSIONS}

In summary, we analyzed the effects of both in-plane and out-of-plane Zeeman fields on the BCS-BEC evolution of a Fermi gas with an ERD spin-orbit coupling field at zero temperature. Depending on the combination of these gauge fields, we found gapless superfluid phases that can be distinguished with respect to the topology of their zeros. For instance, for the BCS-like uniform superfluid phases with zero center-of-mass momentum (where $\Delta_{\mathbf{Q}}=\Delta_{0}$ ), the zeros may correspond to one or two doubly degenerate spheres, two or four spheres, two or four concave spheroids, or one or two doubly degenerate circles. We noted that these phases are distinct because even though their order parameters all have the same $s$-wave symmetry, changes in the topology leave strong evidence on some of the thermodynamic quantities, signaling a topological quantum phase transition.

We also studied the possibility of FFLO-like nonuniform (spatially modulated) superfluid phases with finite center-ofmass momentum and obtained an even richer phase diagram. In particular, we found that while FFLO-like phases are not favored against the BCS-like ones in the absence of an in-plane Zeeman field, even the simplest FFLO-like phases (where the superfluid order parameters involve only a single center-ofmass momentum $\mathbf{Q}$ in such a way that $\left.\Delta_{\mathbf{Q}}=\left|\Delta_{0}\right| e^{i \mathbf{Q} \cdot \mathbf{R}}\right)$ are strongly favored in the absence of an out-of-plane Zeeman field. Therefore, there is a clear competition between outof-plane and in-plane Zeeman fields when both fields are present, and it turns out that the effects of in-plane field rapidly dominate over the out-of-plane one, as a result of which even the simplest FFLO-like phases occupy almost the entire phase diagram. We also noted that allowing multiple center-of-mass momenta, e.g., $\Delta_{\mathbf{Q}}=\left|\Delta_{0}\right| \cos (\mathbf{Q} \cdot \mathbf{R})$, is not expected to favor and stabilize the FFLO-like phases much more than the single- $\mathbf{Q}$ case discussed in this paper. In addition, we further classified the FFLO-like phases with respect to the topology of the momentum-space regions with zero excitation energy.

To conclude, our results suggest that cold atom systems with ERD coupling and in-plane Zeeman field are some of the best candidates for studies on exotic BCS-like gapless uniform and FFLO-like nonuniform superfluid phases, with a greater premise on a route toward studying them under highly controllable atomic settings. As a final remark, it is worth emphasizing that these phases can easily be distinguished by directly measuring their momentum distributions, which is routinely done in every cold atom system via simply looking at their time-of-flight images.

Note added: Recently, there appeared a number of other works on related problems [33].

\section{ACKNOWLEDGMENTS}

This work is supported by the Marie Curie IRG (FP7-PEOPLE-IRG-2010-268239), TÜBİTAK Career (3501110T839), TÜBA-GEBİ, and the National Center for High Performance Computing of Turkey (UHeM-1001232011).
[1] M. Z. Hasan and C. L. Kane, Rev. Mod. Phys. 82, 3045 (2010).

[2] X.-L. Qi and S.-C. Zhang, Rev. Mod. Phys. 83, 1057 (2011).

[3] X.-G. Wen, arXiv:1210.1281.

[4] Y.-J. Lin et al., Nature (London) 471, 83 (2011).

[5] J.-Y. Zhang, S.-C. Ji, Z. Chen, L. Zhang, Z.-D. Du, B. Yan, G.-S. Pan, B. Zhao, Y.-J. Deng, H. Zhai, S. Chen, and J.-W. Pan, Phys. Rev. Lett. 109, 115301 (2012).

[6] P. Wang, Z.-Q. Yu, Z. Fu, J. Miao, L. Huang, S. Chai, H. Zhai, and J. Zhang, Phys. Rev. Lett. 109, 095301 (2012).

[7] L. W. Cheuk, A. T. Sommer, Z. Hadzibabic, T. Yefsah, W. S. Bakr, and M. W. Zwierlein, Phys. Rev. Lett. 109, 095302 (2012).

[8] H. Zhai, Int. J. Mod. Phys. B 26, 1230001 (2012).

[9] J. P. Vyasanakere and V. B. Shenoy, New J. Phys. 14, 043041 (2012).

[10] L. Jiang, X.-J. Liu, H. Hu, and H. Pu, Phys. Rev. A 84, 063618 (2011).

[11] S. Takei, C.-H. Lin, B. M. Anderson, and V. Galitski, Phys. Rev. A 85, 023626 (2012).
[12] M. Gong, S. Tewari, and C. Zhang, Phys. Rev. Lett. 107, 195303 (2011); M. Gong, G. Chen, S. Jia, and C. Zhang, ibid. 109, 105302 (2012).

[13] M. Iskin and A. L. Subaşi, Phys. Rev. Lett. 107, 050402 (2011).

[14] M. Iskin and A. L. Subaşi, Phys. Rev. A 84, 043621 (2011).

[15] W. Yi and G.-C. Guo, Phys. Rev. A 84, 031608(R) (2011).

[16] K. Seo, L. Han, and C. A. R. Sá de Melo, Phys. Rev. A 85, 033601 (2012); Phys. Rev. Lett. 109, 105303 (2012).

[17] K. Zhou and Z. Zhang, Phys. Rev. Lett. 108, 025301 (2012).

[18] R. Liao, Y. Y.-Xiang, and W.-M. Liu, Phys. Rev. Lett. 108, 080406 (2012).

[19] J. Zhou, W. Zhang, and W. Yi, Phys. Rev. A 84, 063603 (2011).

[20] L. He and X. G. Huang, Phys. Rev. Lett. 108, 145302 (2012); Phys. Rev. A 86, 043618 (2012).

[21] M. Iskin, Phys. Rev. A. 85, 013622 (2012).

[22] G. Moore and N. Read, Nucl. Phys. B 360, 362 (1991).

[23] G. Volovik, The Universe in a Helium Droplet (Oxford University Press, New York, USA, 2003).

[24] N. Read and D. Green, Phys. Rev. B 61, 10267 (2000). 
[25] S. Tewari, S. Das Sarma, and D.-H. Lee, Phys. Rev. Lett. 99, 037001 (2007).

[26] T. Mizushima, M. Ichioka, and K. Machida, Phys. Rev. Lett. 101, 150409 (2008).

[27] L. Fu and C. L. Kane, Phys. Rev. Lett. 100, 096407 (2008).

[28] Y. Oreg, G. Refael, and F. von Oppen, Phys. Rev. Lett. 105, 177002 (2010).

[29] J. Alicea et al., Nature Phys. 7, 412 (2011).

[30] J. D. Sau, R. M. Lutchyn, S. Tewari, and S. Das Sarma, Phys. Rev. Lett. 104, 040502 (2010).
[31] P. Fulde and R. A. Ferrell, Phys. Rev. 135, A550 (1964).

[32] A. I. Larkin and Y. N. Ovchinnikov, Zh. Eksp. Teor. Fiz. 47, 1136 (1964) [Sov. Phys. JETP 20, 762 (1965)].

[33] V. B. Shenoy, arXiv:1211.1831; F. Wu, G.-C. Guo, W. Zhang, and W. Yi, Phys. Rev. Lett. 110, 110401 (2013); K. Seo, L. Han, and C. A. R. Sá de Melo, arXiv:1211.5133; X.-J. Liu and $\mathrm{H}$. $\mathrm{Hu}$, arXiv:1302.0553; L. Dong, L. Jiang, and H. Pu, arXiv:1302.1189; X.-F. Zhou, G.-C. Guo, W. Zhang, and W. Yi, Phys. Rev. A 87, 063606 (2013). 